\title{
Prospective and Retrospective Analysis of Gestational Trophoblastic Disease over a Period of 5 Years
}

Nisha Singh, Uma Singh, Sanghmitra Srivastava

\begin{abstract}
Objective: To study incidence and outcome of gestational trophoblastic disease and its variants and changes in gestational trophoblastic neoplasia (GTN) outcome since the use of FIGO 2002 scoring system.
\end{abstract}

\begin{abstract}
Materials and method: A prospective and retrospective cohort study was conducted on all cases of GTD. Those admitted from J an 2005 to Dec 2007 were retrospectively analyzed from hospital records. Cases admitted from J an 2008 to Dec 2009 were followed prospectively. Data was analyzed in terms of methods of diagnosis, FIGO score, treatment methods, success and follow- up feasibility. Statistical analysis was done on SPSS 11 of Windows 2003.
\end{abstract}

Results: Forty-four patients of GTD were analyzed, 21 retrospectively and 23 prospectively. The incidence of GTD was 1.1 per 1,000 admissions and 1.5 per 1,000 deliveries. GTN constituted $1.44 \%$ of all the gynecological cancer cases admitted in 5 years. Invasive mole constituted $68 \%$ of GTN. Seventyone percent of the GTN belonged to stage 1 and $60 \%$ had low risk score. P rospective cases managed according to new FIG O (2002) scoring system showed a faster decline in $\beta$ hCG, lower drug toxicity and higher complete cure rates.

Conclusion: Management of GTN according to new scoring system results in high cure rates. Centralized registry can achieve $100 \%$ follow-up and higher survival rates.

Keywords: H mole, Invasive mole, GTN, Choriocarcinoma, $\beta h C G$.

How to cite this article: Singh $N$, Singh $U$, Srivastava S. Prospective and Retrospective Analysis of Gestational Trophoblastic Disease over a Period of 5 Years. J South Asian Feder Obst G ynae 2013;5(1):11-14.

\section{Source of support: Nil}

\section{Conflict of interest: None}

\section{INTRODUCTION}

Gestational trophoblastic disease (GTD) is an umbrella term for a group of pregnancy-related disorders arising from abnormal placental trophoblast cells. It encompasses a spectrum of interrelated conditions which can be premalignant or malignant. Premalignant forms include $\mathrm{H}$. mole which can be partial or complete while malignant forms gestational trophoblastic neoplasia (GTN) include invasive mole (IM), choriocarcinoma (CC) and placental site trophoblastic tumor (PSTT). All these tumors have varying propensities for local invasion and metastasis. All forms of GTD produce $\beta$ hCG which therefore acts as a tumor marker. Serum $\beta$ hCG levels correlate with disease volume and hence monitoring its levels is used as an accurate biomarker for diagnosis, prognosis and follow-up of GTD.
A mong all GTD, $\mathrm{H}$ mole is the commonest form occurring in 1 in 714 pregnancies in UK. ${ }^{1}$ B ased on thorough pathological review, the incidence of complete and partial mole was found to be 1 in 1,945 and 1 in 6952 pregnancies respectively. A bout $8 \%$ of $\mathrm{H}$ moles transform into GTN. GTN can be a significant cause of morbidity, loss of fertility and rarely mortality in young women if not managed in time. If managed, this is among the rare human malignancies that can be cured even in the presence of widespread dissemination. GTN is highly treatable due to its remarkable sensitivity to chemotherapy. A fter achieving remission with chemotherapy, patients with GTN can anticipate normal reproduction in future.

The successful outcome for patients with GTD has been greatly facilitated by establishment of a centralized registration and treatment system in UK which has enabled the development of effective management policies. The introduction of new FIGO (2002) scoring system has changed the management protocols for the disease. The new system came into practice at our institute since J an 2007. This study was therefore planned to study GTD in terms of its incidence, diagnostic methods, treatment protocols, success and follow-up prior to and after introduction of FIGO (2002) scoring system.

\section{AIMS AND OBJECTIVES}

1. To find the incidence and outcome of GTD and its variants.

2. To evaluate change in the cure rate of GTN after FIGO 2002 scoring system.

\section{MATERIALS AND METHODS}

The study was conducted in the department of Obstetrics and Gynecology, CSM M U , L ucknow, from J an 2007 to Dec 2009.

\section{Design}

It was an analytical prospective and retrospective cohort study.

\section{Subjects}

Total 44 cases of GTD admitted between J an 2005 to D ec 2009. The prospective cohort from Jan 2008 to Dec 2009 included 23 cases and the retrospective cohort from J an 2005 to Dec 2007 included 21 cases of GTD.

\section{Study Protocol}

\section{Prospective Study}

All 23 cases of GTD were followed up from diagnosis to treatment and follow-up till Dec 2009. Data was recorded about 


\begin{tabular}{lcccc}
\multicolumn{5}{c}{ Table 1: Year-wise distribution of all cases of GTD } \\
\hline Years & $\begin{array}{l}\text { Total no. } \\
\text { of cases }\end{array}$ & $\begin{array}{l}\text { Number } \\
\text { of benign } \\
\text { cases }\end{array}$ & $\begin{array}{l}\text { Number } \\
\text { of } \\
\text { malignant } \\
\text { cases }\end{array}$ \\
\hline Retrospective & 2005 & 11 & 08 & 13 \\
& 2006 & 07 & & \\
Prospective & 2007 & 03 & & 09 \\
& 2008 & 09 & 14 & 09 \\
\hline Total & 2009 & 14 & & 22 \\
\hline
\end{tabular}

\begin{tabular}{llll}
\multicolumn{4}{c}{ Table 2: Demographic characteristics of GTD cases } \\
\hline Characteristics & & $\mathrm{n}$ & $\%$ \\
\hline Age & Range & $18-45$ yrs \\
& Mean & 27 yrs & \\
Parity & P $~$ & 7 & 15 \\
& P 1-4 & 30 & 70 \\
Religion & P 5 and above & 7 & 15 \\
& Hindus & 37 & 82 \\
& Muslims & 08 & 18 \\
\hline
\end{tabular}

the diagnostic methods, FIGO scoring, treatment methods, success and follow-up compliance.

\section{Retrospective Study}

Hospital case records of all 21 cases of GTD were reviewed and data recorded in terms of methods of diagnosis, WHO scoring, treatment methods, cure rate and follow-up compliance.

\section{Analysis}

The data collected from prospective and retrospective cohorts was analyzed to find the incidence and cure rates of all variants of GTD. Data was also analyzed to compare type of chemotherapy and success rate of GTN before and after new FIGO (2002) scoring system. Statistical analysis was done on SPSS 11 of W indows 2003.

\begin{tabular}{lc}
\multicolumn{2}{c}{ Table 3: Diagnostic criteria for GTN cases } \\
\hline Criteria & No. of cases \\
\hline Rising hCG & 04 \\
Plateau hCG & 05 \\
Positive hCG after 4 months & 06 \\
Metastasis & 02 \\
Histopathology & 01 \\
Imaging & 04 \\
\hline
\end{tabular}

\section{RESULTS}

The incidence of GTD was 1.1 per 1,000 admissions and 1.5 per 1,000 deliveries. GTN constituted $1.44 \%$ of all the gynecological malignancies admitted in 5 years. O ut of 44 GTD cases, $22(50 \%)$ were benign and $22(50 \%)$ were malignant. Table 1 shows the year wise distribution of all benign and malignant cases in the two cohorts. Table 2 shows the demographic characteristics of all GTD cases.

A mong the benign cases, 21 were complete moles and one was partial mole. USG was the diagnostic modality in $68 \%$ of GTD at mean gestational age of 15 weeks. S. $\beta$ hCG values ranged from 0.50 to $14,54,800 \mathrm{IU} / \mathrm{I}$ with mean value of $9,50,978.328 \mathrm{IU} / \mathrm{I}$. Theca lutein cysts were seen in two (9.1\%) cases. Thyroid profile was done in five with one showing hyperthyroidism. Chest X-ray was done in seven cases, all had normal findings.

Suction evacuation was done in $20(90 \%)$ cases. One 45-year-old P9+1with complete mole was treated with hysterectomy and a twin with partial mole had cesarean at 28 weeks for APH respectively. A djuvant therapy included repeat curettage (2), blood transfusion (5) and prophylactic chemotherapy (4). Histopathology reports were available in only $10(45.4 \%)$ prospective cases which confirmed $\mathrm{H}$ mole. All 22 benign GTD showed a decline in S. $\beta$ hCG value after treatment. S. $\beta$ hCG follow-up was done in all 14 prospective cases till complete cure but only single post-treatment S. BhCG record was available in eight retrospective cases.

Table 3 shows the different diagnostic criteria used for 22 GTN cases. $\mathrm{H}$ mole was the most common preceding event seen in $72 \%$ cases of GTN followed by abortion in $18.1 \%$ and normal pregnancy in $9.09 \%$. M ean S. $\beta$ hCG was $2,64,777.88$

\begin{tabular}{lccc}
\multicolumn{2}{c}{ Table 4: Comparison of outcome of chemotherapy in low score $(n=9)$ and high score $(n=11)$ GTN cases } \\
\hline Chemotherapy in low score cases & No. of cases treated & No. of course/dose given & Outcome of treatment \\
\hline Methotrexate & 01 & 1 dose & Not known \\
Methotrexate & 01 & 2 doses & Not known \\
Methotrexate & 03 & 1 course & Not known \\
Methotrexate & 01 & 3 courses & Treated \\
Methotrexate & 02 & 4 courses & Treated \\
Methotrexate & 01 & 6 courses & Treated \\
Chemotherapy in high & & & Refused further \\
score cases & 1 & 1 & treatment \\
MAC & & 2 & Cured \\
MAC & 2 & $1+2$ & Cured \\
Mtx followed by MAC & 1 & 5 & Cured \\
EMA-CO & 1 & 6 & Cured \\
EMA-CO & 3 & 8 & Cured \\
EMA-CO & 1 & $3+3$ and $6+4$ & Cured \\
Mtx-EMA-CO & 2 & &
\end{tabular}


Prospective and R etrospective Analysis of Gestational Trophoblastic Disease over a Period of 5 Y ears

\begin{tabular}{lccc}
\hline \multicolumn{4}{c}{ Table 5: Comparison of outcome of retrospective $(n=9)$ and prospective GTN cases $(n=9)$} \\
\hline Chemotherapy in retrospective cases & No. of cases & No. of courses/doses given & Treatment outcome \\
\hline Methotrexate (Mtx) & 4 & $1,2,1,1$ & Not known \\
MAC & 1 & 1 & Not known \\
Mtx + MAC & 2 & $2+2,2+1$ & Treated \\
EMA-CO & 2 & 5,8 & Treated \\
\hline Chemotherapy in prospective cases & & & Treated \\
\hline Methotrexate & 4 & $4,3,4,1$ & Treated \\
EMA-CO & 3 & $6,6,6$ & Treated \\
Mtx + MAC +EMA-CO & 1 & $1+3+3$ & Treated \\
Mtx + EMA-CO & 1 & $2+4$ &
\end{tabular}

IU/I. FIGO staging of GTN cases showed 13 in stage 1 ; six in stage 2; two in stage 3 and one in stage 4. Classification of GTN showed that 15 (68.18\%) were invasive mole, five $(22.7 \%)$ gestational choriocarcinoma and two $(0.09 \%)$ PSTT. A ccording to FIGO (2002) scoring system, $16(72.7 \%)$ cases had high risk scores ( 7 or above) and six $(27.3 \%)$ had low risk score (0-6).

Table 4 shows the comparative outcome of chemotherapy in low risk and high risk cases. Single agent chemotherapy with methotrexate was used in nine $(40.9 \%)$ cases and multiagent chemotherapy with MAC, EMA-CO or methotrexate-EM A regimen was used in $11(59.1 \%)$ cases. Table 5 shows the comparative outcome of retrospective and prospective cases. Four of the retrospective cases could not be treated due to financial constraints. A djuvant treatment included hysterectomy ( 5 cases) and blood transfusion (7 cases). Nine $(100 \%)$ prospective and five (38.46\%) retrospective cases were completely cured. Five were lost to follow-up after complete therapy and three discontinued treatment due to cost factor.

The two PSTT cases were both retrospective. O ne 20-year nullipara had $\mathrm{H}$ mole treated by suction and histopathology confirmed CHM. S. $\beta$ hCG decreased from 4,69,100 to 3221 IU/I but USG showed a lesion $1.7 \times 1.2 \mathrm{~cm}$ infiltrating uterine wall. A fter first course of methotrexate, she was lost to followup. The other was a 37years old P3+2 with induced septic abortion in A R F who received three hemodialysis and then was referred to us. The MRI was suggestive of PSTT and single hCG after 6 weeks of abortion was 635 IU/l. The patient left without treatment.

\section{DISCUSSION}

$\mathrm{H}$. mole is the commonest form of GTD occurring in 1 in 714 pregnancies in UK. ${ }^{1}$ Incidence of GTD was 1.1 per 1,000 admissions and 1.5 per 1,000 deliveries which is slightly higher. O ut of total 44 GTD cases, 22 (50\%) were benign and 22 (50\%) were malignant showing a significant load of GTD with almost $50 \%$ cases being malignant.

A liza et $\mathrm{al}^{3}(2006)$ stated that most of the first trimester studies of complete moles demonstrate a typical appearance but in cases of partial moles USG characteristics may be difficult to be identified prior to second trimester. In this study, USG had diagnosed $68 \%$ of cases at mean gestational age of 15 weeks. In study by Soto-W right et al ${ }^{4}$ incidence of theca Iutein cysts was $9 \%$ while there were no cases with hyperthyroidism and X -ray chest abnormalities. In the present study too, theca lutein cysts were seen in $9.1 \%$ cases and normal $X$-ray findings were seen in all cases investigated for pulmonary abnormalities. Hyperthyroidism was found in one out of only five cases investigated. The lower incidence of all these additional findings once thought to be classical medical complications related to $\mathrm{H}$ mole is due to early detection by USG. All forms of GTD produce $\beta$ hCG which therefore acts as a tumor marker. The mean $\beta \mathrm{hCG}$ value was as high as 9,50,978.328 IU/l.

RCOG $(2004)^{5}$ and A liza et $\mathrm{al}^{3}$ (2006) recommended suction evacuation as the treatment of choice in molar pregnancies. O ur centre showed a post suction decline of $\beta$ hCG in all cases. Tidy et $a l^{6}$ deferred use of medical methods for treatment as it was associated with higher rate of chemotherapy related complications. In the present study, prophylactic chemotherapy was given in one patient who came after medical abortion and did not have any complications. $\mathrm{H}$ mole was the most common preceding event for GTN (72\%) followed by abortion (18.1\%) and normal pregnancy $(9.09 \%)$ which is in accordance with review article by A liza et al. ${ }^{3}$

Criteria laid by The FIGO council 2000 for diagnosis of persistent GTN include rise or plateau $\beta$ hCG titers after evacuation or persistently detectable $\beta$ hCG titers even 6 months post evacuation or histologic diagnosis of choriocarcinoma. In the present study, persistent GTN was diagnosed by detectable $\beta$ hCG after 4 months, plateau $\beta$ hCG titers, rising BhCG titers, USG/MRI lesion, metastasis and HPE.

Osborne et $\mathrm{al}^{7}$ and FIGO (2002) have suggested single agent chemotherapy (methotrexate) for low risk and combination therapy for high risk cases as the treatment of choice in GTD. In this study, nine low risk cases were treated by single agent chemotherapy (methotrexate) and 11 high risk cases were treated by multiagent chemotherapy (M A C, EM A $\mathrm{CO}$ and methotrexate followed by EM A-CO regimens).

Study by Newlands et $\mathrm{al}^{8}$ has shown EMA-CO as the treatment of choice in high risk cases with improved primary response rate and lower toxicity rates as compared to MAC regimen used previously. In this study, also it was observed that in cases of high-risk GTN, mean decline in $\beta$ hCG value was more, drug toxicity was less and cure rates were high in prospective group managed according to new FIGO (2002) scoring systems and given EMA-CO as compared to retrospective group treated with $M A C$ regime. 
On comparing the outcome of nine prospective cases of GTN managed according to new FIGO (2002) scoring system with 13 retrospective cases managed according to WHO scoring system, it was observed that although the first $\beta$ hCG levels decreased in both groups mean decline in $\beta$ hCG value was more in prospective group. Less drug toxicity due to chemotherapy and higher complete cure rates were observed in cases of prospective as compared to retrospective group. The improved outcome in the prospective group is also attributed to the availability of chemotherapy drugs free of cost to the patients since, last few years which has greatly improved the compliance and follow-up rates.

\section{CONCLUSION}

There is a significant load of GTN in our population with nearly $50 \%$ being malignant. Almost $100 \%$ success rate is achieved with latest protocols. Centralized registry must be done in OPD to get complete follow-up data and to achieve $100 \%$ survival.

\section{ACKNOWLEDGMENTS}

The data analysis and manuscript preparation were done by DrNisha Singh. Dr Sanghmitra collected the data and reviewed the literature, while Dr U ma Singh gave valuable suggestions in the manuscript.

\section{REFERENCES}

1. Tham BWL, Everard JE, Tidy JA, Drew D, Hancock BW. Gestational trophoblastic disease in the A sian population of Northern England and North Wales. BJ OG 2003;110:555-59.

2. Berkowitz RS, G oldstein DP. Gestational trophoblastic disease in berek and N ovak's gynecology (15th ed). South A sian B erek IS (Ed). LWW publication 2012.p 1459.

3. Leiser $A L, C$ arol. Evaluation and management of gestational trophoblastic disease. Commun Oncol 2006 M arch:152-55.
4. Soto-W rightV , B ernstein M, Goldstein DP, B erkowitz RS. The changing clinical presentation of complete molar pregnancy. Obstet Gynecol 1995;86:775-79.

5. M anagement of Gestational Trophoblastic Neoplasia, Royal College of Obstetricians and Gynaecologists (RCOG); 2004 Feb. revised 2010 F eb 11 p. (Green-top Guideline; no. 38).

6. Tidy JA, Gillispie A M , B right N, Radstone CR, Coleman RE, Hancock BW. Gestational trophoblastic disease: A study of mode of evacuation and subsequent need for treatment with chemotherapy. Gynecol Oncol 2000;78(3pt 1):309-12.

7. Osborne $R$, Gerulath $A$. W hat is the best regimen for low risk gestational trophoblastic neoplasia? A review. J Reprod Med 2004;49:602-16.

8. Newlands ES, Bagshawe KD, B egent RH, Holden L. Results with the EMA/CO (etoposide, methotrexate, actinomycin D, cyclophosphamide, vincristine) regimen in high risk gestational trophoblastic tumors 1979 to 1989. Br J Obstet Gynecol 1991;98:550-57.

\section{ABOUT THE AUTHORS}

\section{Nisha Singh}

Professor, Department of Obstetrics and Gynecology, CSM M edical University, Lucknow, Uttar Pradesh, India

CorrespondenceAddres A 172 South City, Raebareli Road, Lucknow Uttar Pradesh, India, Phone: 9415588458, e-mail: nisha.kgmc@ gmail.com

\section{Uma Singh}

Professor, D epartment of Obstetrics and Gynecology, CSM M edical University, L ucknow, Uttar Pradesh, India

\section{Sanghmitra Srivastava}

Postgraduate Student, Department of O bstetrics and G ynecol ogy, CSM M edical U niversity, L ucknow, U ttar Pradesh, India 